\title{
Aspergilosis broncopulmonar alérgica
}

\author{
Benjamín Ramírez Cisneros ${ }^{1}$ \\ Dr. Giovanni Sedó Mejía ${ }^{2}$
}

\footnotetext{
${ }^{1}$ Médico General. Universidad de Costa Rica Hospital San Juan de Dios. Benramcis@gmail.com

${ }^{2}$ Médico Especialista en Medicina Interna y Alergología, Unidad 3, Medicina Interna. Hospital San Juan de Dios. Profesor de la Cátedra de Medicina Interna UCR.
}

\section{RESUMEN}

La Aspergilosis Broncopulmonar Alérgica es una entidad clínica que se presenta en pacientes asmáticos y portadores de fibrosis quística con transfondo atópico importante. Dicha enfermedad es producto de un fenómeno de inflamación pulmonar secundaria a una respuesta inmune compleja montada en un huésped colonizado por Aspergillus sp. La historia natural y estadios de la enfermedad son complejos y se definen clínicamente y según su respuesta a tratamiento. El diagnóstico se realiza basado en clínica, radiología y pruebas de laboratorio. El tratamiento se basa en corticoesteroides orales y antifúngicos como ahorradores de esteroides.

\section{PALABRAS CLAVE}

Aspergilosis Broncopulmonar Alérgica, hipersensibilidad tipo I y III, asma grave, Aspergillus spp.

\begin{abstract}
Allergic bronchopulmonary aspergillosis is a clinical entitiy which typically affects asthmatic and cystic fibrosis patients with an important allergic predisposition. The disease is a pulmonary inflammatory phenomenon due to a complex immune response against the colonization of Aspergillus sp. The clinical stages and natural history of this disease are complex and are defined clinically and by the patient's response to treatment. The diagnosis is made on clinical, radiological and laboratory findings. The treatment is based on oral corticosteroids and antifungal therapy.
\end{abstract}

\section{KEY WORDS}

Allergic Bronchopulmonary Aspergillosis, hypersensitivity type I and III, severe asthma, Aspergillus spp.

\section{INTRODUCCIÓN}

El asma es una enfermedad muy prevalente en Costa Rica, de acuerdo con el Estudio Internacional de Asma y Alergías en la Niñez (ISAAC, por su siglas en inglés), un $26,9 \%$ de niños de 6-7 años costarricenses tenían el diagnóstico médico de asma ${ }^{(1)}$.

Se cree que hasta el $10 \%$ de la población se encuentra pobremente controlada a pesar de utilizar las terapias combinadas de corticoesteroides inhalados a altas dosis, más beta dos agonistas de larga duración, es decir pacientes con asma grave, ocasionando un mayor impacto en la calidad de vida. De un tercio hasta la mitad de estos pacientes con asma grave tienen sensibilización atópica a hongos filamentosos, sobre todo Aspergillus fumigatus. La evidencia estableció que la sensibilización a hongos se asocia a un fenotipo de asma más grave. En consecuencia, emergió un subgrupo importante identificable como asma grave con sensibilización a hongos (SAFS). La identificación como un fenotipo reconocible de asma parece acarrear implicaciones terapéuticas importantes ${ }^{(2)}$.

El Aspergillus es un género de hongos con ubicación en la tierra y vegetación ${ }^{(3)}$, existen aproximadamente 250 especies de Aspergillus, pero solo pocas especies son patógenas para el ser humano ${ }^{(4)}$. 
La aspergilosis broncopulmonar alérgica (ABPA) es una entidad clínica caracterizada por inflamación pulmonar causada por hipersensibilidad a Aspergillus fumigatus, que se presenta en paciente típicamente asmáticos o portadores de fibrosis quística (FQ) ${ }^{(3)}$.

El hongo asociado a ABPA es el Aspergillus fumigatus, sin embargo, se han descrito otras especies de hongos (Candidad, Penicillinum) que son capaces de producir micosis alérgicas broncopulmonares entidades similares al ABPA (3).

El Aspergillus fumigatus se caracteriza por estar relacionado a distintas entidades clínicas respiratorias, entre ellas se encuentran la aspergilosis invasiva, aspergiloma, rinitis y asma alérgica mediada por IgE, neumonitis por hipersensibilidad, neumonía crónica necrotizante y la $\operatorname{ABPA}^{(3)}$.

La ABPA es una entidad clínica que suele cursar sin diagnóstico y si cursa sin tratamiento produce complicaciones irreversibles $^{(4)}$ por lo que se realiza una revisión de tema de la epidemiología, patogénesis, características clínicas y tratamiento de dicha enfermedad.

\section{EPIDEMIOLOGÍA}

La ABPA ocurre prácticamente sólo en pacientes asmáticos y portadores de FQ con transfondo atópico importante ${ }^{(5)}$.

La sensibilización a Aspergillus (SA) se define por la presencia de una respuesta inmediata a la prueba de sensibilidad cutánea ${ }^{(2)}$. No todos los pacientes con SA ni SAFS cursan con ABPA, sin embargo, es condición sine qua non para el desarrollo a $\mathrm{ABPA}^{(4)}$.

Según varios estudios prospectivos, la prevalencia de SA ronda entre 5,5-38,5\%. La prevalencia de ABPA ha sido reportada desde un 2,5-22,3\%, la prevalencia aumenta si el estudio es realizado en un centro de referencia de patología pulmonar ${ }^{(2)}$. Un estudio estima que a nivel mundial deben haber 4,8 millones de pacientes con ABPA con una población mundial asmática de 193 millones ${ }^{(2)}$.

Aunque puede presentarse en cualquier edad, la mayoría de las veces debuta en pacientes entre la tercera y cuarta década ${ }^{(3)}$. No se ha descrito predilección por algún género ${ }^{(6)}$.
En este milenio se han realizado algunos estudios prospectivos, analizando la prevelanecia de SA y de ABPA en pacientes asmáticos. La prevalencia de ABPA reportada en Nueva Zelanda fue de $4,9 \%$, en Arabia Saudita se reportó un 2,7\%, múltiples estudios realizados en India han reportado desde un $6,9-22,3 \%$ y un estudio realizado en China reportó $2,5 \%{ }^{(7)}$.

En Costa Rica, se desconoce la prevalencia de ABPA. Sin embargo, se realizó un estudio para determinar la prevalencia de SA en pacientes asmáticos severos en el Hospital San Juan de Dios, y se determinó que un $18 \%$ de estos pacientes presentaron la prueba cutánea positiva (8)

Es importante recalcar que la prevalencia de ABPA aumenta al considerarse la población asmática internada con el diagnóstico de asma severa ${ }^{(4)}$. Por lo que se debe de considerar la ABPA dentro de los diagnósticos diferenciales del asma severa o asma con respuesta tórpida al tratamiento convencional.

\section{PATOGÉNESIS}

La patogénesis de la ABPA es compleja, sin embargo, se conoce el importante rol de la respuesta inmunológica, principalmente alérgica, en la patogenia de esta entidad clínica. Es importante recalcar que la ABPA es una respuesta de hipersensibilidad ante los antígenos expresados secundarios a la colonización del hongo en la vía aérea inferior ${ }^{(3 ; 5)}$ y no se trata de una infección del hongo como tal.

La ABPA se da en el contexto de un paciente atópico que ante la presencia de Aspergillus fumigatus a nivel pulmonar se lleva a cabo una activación de leucocitos con la liberación subsecuente de citoquinas e inmunoglobulinas que desencadenan un proceso inflamatorio $\operatorname{pulmonar}^{(5)}$.

La evidencia microbiológica y patológica ha demostrado que la hipersensibilidad en la ABPA no es únicamente de tipo I (mediado por IgE). Estudios han encontrado también complejos inmunes circulando en pacientes con el diagnóstico de ABPA (hipersensibilidad de tipo III) ${ }^{(9)}$.

Las esporas del hongo son pequeñas $(2-3 \mu \mathrm{m})$ por lo que son fácilmente inhaladas y se depositan en la vía aérea inferior $^{(5)}$, si el 
ambiente $\mathrm{y}$ huésped lo permiten el hongo proliferará, produciéndose colonización del hongo ${ }^{(3)}$. Una vez que el hongo prolifera, las esporas que germinan junto con las hifas, hacen que aumente la carga de antígenos ${ }^{(2)}$. Las células presentadoras de antígenos procesan estos antígenos fúngicos por medio de receptores inmunológicos innatos como el receptor tipo Toll, e inician la secreción de moléculas (como TSLP, OX40L, CCL17 e IL-33) que reclutan y favorecen la proliferación y diferenciación de linfocitos Th2 ${ }^{(2)}$.

En un paciente no susceptible y sin inmunocompromiso, montará una respuesta Th1 que prevendría una infección y colonización por el hongo ${ }^{(5)}$. En un huésped susceptible, con mayor respuesta Th2, se llevará a cabo la activación de esta vía, que involucra Il-4, Il-5, la producción de IgE y reclutamiento de células como los mastocitos y eosinófilos ${ }^{(5)}$. El CCL17 también atrae linfocitos $\mathrm{T}$ regularadores, células capaces de suprimir la respuesta Th1 y de macrófagos, favoreciendo la colonización del hongo $^{(2)}$.

Al unirse los anticuerpos IgE específicos a los mastocitos, a la siguiente exposición de antígenos del hongo, se llevará a cabo una reacción de hipersensibilidad ${ }^{(2)}$.

La activación de estas células inmunes es lo que se ha descrito como el evento culpable de la respuesta inflamatoria que se observa en pacientes con ABPA.

Sin embargo, es importante recalcar que se ha descrito en la bibliografía que hasta un $25 \%$ de los pacientes asmáticos se clasifican como SA, pero solo una pequeña fracción presentan clínicamente $\mathrm{ABPA}^{(5)}$, por lo que deben existir más mecanismos fisiopatológicos aún no descritos o bien una susceptibilidad genética para que el paciente desarrolle ABPA propiamente dicha.

Se han realizado estudios genéticos para estudiar defectos de inmunidad innata y adaptativa para intentar explicar la susceptibilidad del huésped (7). Estos defectos son los que se han postulado como posible explicación al porqué los huéspedes susceptibles montan una mayor respuesta Th2 (que favorece colonización e hipersensibilidad) versus una respuesta Th1 (que favorece la eliminación del hongo), fenómeno inmunológico típico de pacientes atópicos.

\section{PRESENTACIÓN CLÍNICA}

La ABPA típicamente se presenta en pacientes con historia de asma de difícil control o asma severa ${ }^{(9)}$. La presentación clínica de la ABPA incluye exacerbaciones asmáticas recurrentes, sibilancias, tos e infiltrados radiológicos, aunados a sintomatología sistémica como fiebre, anorexia, cefalea y mal estado general ${ }^{(5)}$.

La sintomatología es muy similar a la del asma y FQ, por lo que se debe de descartar el diagnóstico en todo paciente con asma severa ${ }^{(6)}$. Sin embargo, ciertos hallazgos son útiles para distinguir clínicamente la ABPA. La tos, hasta en un $50 \%$ de los pacientes, es productiva, y es caracterizada por un esputo espeso, de color café-verde, y se observa como tapones de moco espeso ${ }^{(3)}$. Además pueden presentar acropaquias, crépitos y datos de falla cardiaca derecha en estadios avanzados ${ }^{(3)}$.

\section{RADIOLOGÍA}

Los hallazgos radiológicos son fundamentales para el diagnóstico y la sospecha clínica de la ABPA. Los hallazgos no siempre correlacionan con la sintomatología, y hasta en una tercera parte de los pacientes pueden estar asintomáticos y tener infiltrados pulmonares ${ }^{(3)}$.

La radiología se caracteriza por tener infiltrados transitorios, que resuelven a las semanas espontáneamente o posterior a un ciclo de esteroides (3), y bronquiectasias ${ }^{(9)}$. Estas se caracterizan por ser cilíndricas y proximales (centrales), hallazgo fundamental para el diagnóstico diferencial de bronquiectasias. En la cuadro 1 se describen hallazgos radiológicos en ABPA. 
Cuadro 1 Hallazgos radiológicos en ABPA

\section{Frecuentes}

Infiltrados intersticiales intermitentes

Bronquiectasias centrales

Consolidación

Atelectasia lobar

"Dedo en guante"- impactación de moco en bronquios dilatados

"Sombra de ferrovía" - lineales paralelas que se extienden del hilio hacia la distribución bronquial, reflejando edema e inflamación bronquial

"Sombra de pasta de dientes"- impactación de moco en bronquios

"Sombra de anillo"- bronquios dilatados con pared bronquial inflamados

\section{Menos frecuentes}

Bulas

Neumotórax

Derrame pleural

Lesiones nodulares cavitadas

Aspergiloma

Fuente: Mayo Clin Proc, 2001; 76: 930-938.

Una radiografía de tórax normal no excluye el diagnóstico, por lo que se recomienda realizar una tomografía axial computarizada en todo paciente con sospecha de ABPA. El hallazgo de bronquiectasias centrales en un paciente asmático es otro hallazgo que debe levantar sospecha de ABPA ${ }^{(3)}$. La fibrosis y panalización son hallazgos sugestivos de enfermedad terminal y conllevan mal pronóstico ${ }^{(5)}$.

\section{LABORATORIOS}

Varios exámenes de laboratorio y de gabinete son de utilidad diagnóstica y de seguimiento en la ABPA.

Los pacientes con ABPA se presentan con eosinofilia en el hemograma, y se considera un hallazgo inespecífico de actividad de la enfermedad ${ }^{(3)}$. Típicamente se ha utilizado el corte de más de 1000 células/ $\mu 1^{(4)}$ como criterio diagnóstico.

Es necesario documentar una IgE sérica elevada para el diagnóstico de ABPA. Según los criterios diagnósticos, se debe utilizar el corte de 1000 $\mathrm{IU} / \mathrm{ml}$. La IgE es un marcador serológico útil para marcar actividad de enfermedad y respuesta a tratamiento ${ }^{(3)}$. Una $\operatorname{IgE}$ normal en pacientes sintomáticos es un hallazgo muy específico para descartar el diagnóstico de ABPA ${ }^{(3)}$. Importante destacar que la IgE sérica usualmente hace pico cuando más hay infiltrados radiológicos y durante exacerbaciones ${ }^{(3)}$.

Para medir sensibilidad al hongo se realiza una prueba cutánea para Aspergillus fumigatus. Una respuesta inmediata (hipersensibilidad tipo 1 IgE) es altamente sensible pero no específica para ABPA ${ }^{(5)}$. La respuesta IgG tardía es positiva de forma variable, por lo que usualmente no se considera y se desconoce su significancia (4)

La medición de anticuerpos específicos contra Aspergillus fumigatus tanto IgE como IgG es considerado fundamental para el diagnóstico de ABPA. Por medio de la técnica de ELISA se pueden medir anticuerpos IgE específicos $\mathrm{y}$ permiten diferenciar entre SA y ABPA (4). Comercialmente se utiliza principalmente la prueba de ImmunoCap, que es un método muy sensible ${ }^{(7)}$.

Se han descrito antígenos recombinantes de Aspergillus, que son más específicos de ABPA que no se presentan en pacientes con SA ${ }^{(7)}$. Dentro de los alérgenos recombinantes evaluados se encuentran Asp f1, Asp f2, Asp f3, Asp f4 y Asp f6, en pacientes con ABPA se elevan los anticuerpos contra rAsp f4 y rAsp f6 que no se ven en pacientes con $\mathrm{SA}^{(7)}$. Sin embargo, los estudios que han evaluado utilizar estos anticuerpos contra antígenos recombinantes han sido difíciles de reproducir, por lo que aún no se han considerado como un criterio serológico fidedigno para el diagnóstico de $\mathrm{ABPA}^{(7)}$.

Un cultivo de esputo positivo por Aspergillus fumigatus ayuda al dignóstico de colonización o Aspergiloma, pero no es un criterio de $\mathrm{ABPA}^{(4)}$. Al ser un germen ubicuo, los pacientes podrían tener un esputo positivo por Aspergillus fumigatus, sin necesariamente tener SA o ABPA (4).

Las pruebas de función pulmonar muestran una obstrucción de la vía aérea que es, al menos parcialmente reversible, principalmente en ABPA temprana o leve. En cambio, una obstrucción sin reversibilidad y volúmenes pulmonares reducidos debido a cambios intersticiales reflejan una enfermedad avanzada (4). La capacidad de difusión (DLCO) se puede documentar disminuida durante una exacerbación y en estadios avanzados ${ }^{(4)}$. Es importante recalcar que la ABPA se da en 
pacientes con patología pulmonar subyacente, por lo que las pruebas de función pulmonar pueden ser muy variables y podrían únicamente reflejar la patología de fondo (asma o FQ).

\section{DIAGNÓSTICO}

El diagnóstico de la ABPA está basado en hallazgos clínicos, apoyados de estudios serológicos y radiológicos. Sin embargo, existen criterios diagnósticos que permiten ayudar al clínico. Dichos criterios se han ido modificando con el tiempo, siendo los más utilizados los propuestos por Greenberg \& Patterson (Cuadro 2) ${ }^{(9)}$.

Cuadro 2 Criterios diagnósticos de ABPA de Greenberg \& Patterson

\begin{tabular}{l}
\hline ABPA Seropositivo (ABPA-S) \\
Antecedente de Asma (severa o de difícil control) \\
IgE sérico total elevado (usualmente $>1000 \mathrm{IU} / \mathrm{mL}$ ) \\
Respuesta cutánea inmediata positiva para $A$. \\
fumigatus \\
Anticuerpo IgE específico para $A$. fumigatus elevado \\
Presencia de precipitinas séricas o anticuerpo IgG \\
específico para $A$. fumigatus elevados \\
ABPA Bronquiectasias Centrales (ABPA-CB) \\
Los criterios de ABPA-S positivos y \\
Bronquiectasias centrales demostrado por TAC de \\
alta resolución
\end{tabular}

\section{Otros datos}

Eosinofilia en hemograma (usualmente ausentes si paciente toma esteroides)

Infiltrados intermitentes

Expectoración de tapones mucosos cafés

Bronquios impactados con moco demostrado por estudios radiológicos

Cultivo de esputo positivo por A. Fumigatus

Fuente: Fishman's Pulmonary Diseases and

Disorders. Cuarta Edición Mc-Graw Hill Medical, Estados Unidos, Capítulo 49. 837-844

Es importante destacar que existen criterios diagnósticos para dos entidades clínicas en ABPA. La ABPA-S se refiere a un paciente con criterios serológicos positivos pero sin hallazgos tomográficos de bronquiectasias. ABPA-CB se diagnostica en pacientes con los criterios serológicos positivos pero además con cambios radiológicos de bronquiectasias centrales ${ }^{(9)}$.

Los pacientes con ABPA-S tienen menos sintomatología, valores de IgE más bajos, menor obstrucción al flujo aéreo y menos exacerbaciones ${ }^{(9)}$, por lo que se estipula que se podría tratar de una entidad diagnosticada de forma temprana ( $\sin$ cambios estructurales) $o$ leve, y el tratamiento oportuno podría frenar la progresión de la enfermedad ${ }^{(3)}$, sin embargo esto no está del todo claro ni probado.

\section{HISTORIA NATURAL Y ESTADIOS CLÍNICOS}

Se han descrito cinco estadios de enfermedad definidos en el Cuadro 3. Sin embargo, la historia natural de la enfermedad aún no ha sido bien dilucidada, puesto que la enfermedad no necesariamente progresa según los estadios clínicos definidos ${ }^{(3)}$. Los estadios son dinámicos y varían con el tiempo y con el tratamiento. No se ha demostrado, pero se estipula que si se diagnostica de forma temprana $y$ se trata oportunamente, se enlentece o previene el estadio final (estadio V) ${ }^{(3 ; 4)}$.

El estadio I es cuando el paciente está sintomático y se realiza el diagnóstico (ABPA-S sintomática), en este estadio tienen una adecuada respuesta a tratamiento con esteroides orales y entran al estadio II si luego de 6 meses persisten asintomáticos ${ }^{(3 ; 4)}$. El paciente puede permanecer de forma indefinida en este estadio, o tener una exacerbación/recaída y clasificarse en estadio III $^{(3)}$.

Se considera que un paciente está en estadio IV si el paciente a pesar de estar con esteroides de forma continua persiste sintomático. También se considera que el paciente está en este estadio si al retirar el esteroide el paciente se exacerba ${ }^{(3 ; 4)}$. Los pacientes en estadio $\mathrm{V}$, con enfermedad pulmonar fibrótica y tos productiva crónica, usualmente son pacientes asmáticos con infiltrados recurrentes y que han sido tratados con ciclos de antibióticos en múltiples ocasiones por confundir este estadio con una infección pulmonar o por presentar una sobreinfección ${ }^{(3)}$. En este estadio se considera que aún hay actividad inmunológica, por lo que a pesar de estar en un estadio terminal y avanzado, se recomienda mantener la terapia ${ }^{(3)}$.

Dentro de los criterios para determinar el estadio clínico del paciente se encuentran marcadores serológicos y hallazgos radiológicos, por lo que es fundamental estar midiendo la $\operatorname{IgE}$ sérica, realizar hemogramas y controles radiológicos. 
Títulos altos de IgE sérica total y eosinofilia son marcadores de enfermedad activa.

\section{DIAGNÓSTICO DIFERENCIAL}

El diagnóstico diferencial es amplio e incluye asma de difícil control, FQ, tuberculosis, sarcoidosis, neumonía bacteriana, neumonías eosinofílicas, Síndrome de Churg-Strauss, Síndrome de Loeffler, micosis alérgica broncopulmonar entre otras entidades clínicas ${ }^{(3 ;}$ $4 ; 5)$.

\begin{tabular}{l} 
Cuadro 3. Estadios clínicos de ABPA \\
\hline Estadio I: Agudo \\
Síntomas de exacerbación asmática \\
IgE sérica elevada \\
Eosinofilia en hemograma \\
Infiltrados radiológicos \\
fugáces/intermitentes \\
Marcadores serológicos positivos \\
Respuesta a tratamiento \\
\hline Estadio II: Remisión \\
Resolución sintomática \\
Resolución de infiltrados pulmonares \\
Mejoría en marcadores serológicos (IgE, \\
eosinofilia, títulos de anticuerpos) \\
\hline Estadio III: Exacerbación/recurrencia \\
Recurrencia/progreso de síntomatología \\
Infiltradores pulmonares que recurren \\
IgE séricos que aumentan \\
\hline Estadio IV: Asma dependiente de \\
esteroides \\
Asma refractaria dependiente de esteroides \\
IgE séricos persistentemente elevados \\
Marcadores serológicos persistentemente \\
elevados \\
\hline Estadio V: Enfermedad pulmonar fibrótica \\
Asma refractaria dependiente de esteroides \\
Enfermedad fibrótica pulmonar (obstrucción \\
irreversible, patrón restrictivo, alteración de \\
la capacidad de difusión \\
Síntomas de bronquiectasias crónicas \\
(broncorrea, infecciones a repetición \\
\hline Fuente: Fishman’s Pulmonary Diseases and \\
Disorders. Cuarta Edición Mc-Graw Hill \\
Medical, Estados Unidos, Capítulo 49. 837- \\
844
\end{tabular}

\section{TRATAMIENTO}

El tratamiento se basa principalmente en esteroides orales y/o tratamiento antifúngico. La meta del tratamiento es inducir remisión (estadio
II) y disminuir el proceso inflamatorio intentando disminuir cambios estructurales (estadio V) con la menor cantidad de efectos adversos ${ }^{(4)}$.

Los esteroides orales son la piedra angular del tratamiento de la ABPA, suprimen la hipersensibilidad y actúan como antiinflamatorios (4). La respuesta de la enfermedad usualmente es dependiente de la duración y la cantidad de esteroide. Existen varios esquemas recomendados en la bibliografía. Un esquema más agresivo recomendado por algunos autores estipula dar $0,75 \mathrm{mg} / \mathrm{kg}$ de Prednisolona por 6 semanas y luego 6 semanas a $0,5 \mathrm{mg} / \mathrm{kg}$ y cada 6 semanas disminuir la dosis $5 \mathrm{mg}$ para un total de 6-12 meses para el estadio $\mathrm{I}^{(3 ; 4 ; 5)}$.

El agente antifúngico utilizado antiguamente era el ketoconazol, sin embargo fue sustituido por el uso de Itraconazol, siendo este último menos tóxico ${ }^{(4)}$. El principio de dar tratamiento antifúngico a pesar de no tratarse de una infección es intentar disminuir carga antigénica. Se indica en estadios III y IV ${ }^{(3 ; 4 ; 5 ; 6)}$ y se intenta utilizar como ahorrador de esteroides en estadio IV. Un esquema recomendado es por 16 semanas $200 \mathrm{mg}$ dos veces al día y luego 16 semanas 200 mg cada día ${ }^{(3)}$.

No hay suficiente evidencia para apoyar el uso de Omalizumab o esteroides inhalados ${ }^{(3 ; 4 ; 5 ; 6)}$.

Se monitoriza la respuesta al tratamiento con base en la sintomatología, cambios radiológicos, pruebas de función pulmonar y la $\operatorname{IgE}$ sérica total.

\section{CONCLUSIÓN}

La ABPA es una enfermedad que afecta principalmente pacientes atópicos asmáticos $\mathrm{o}$ portadores de FQ. Es un diagnóstico a descartar en asma severa y se debe tamizar en pacientes con FQ. La sintomatología de ABPA es muy similar a la de las patologías pulmonares subyacentes, por lo que se debe de tener alta sospecha diagnóstica.

Se desconoce la prevalencia de ABPA en Costa Rica.

Aún no se conoce la historia natural de la enfermedad con claridad ni se puede predecir la respuesta al tratamiento, por lo que se debe monitorizar al paciente durante el tratamiento 
clínicamente, con pruebas de función pulmonar, pruebas serológicas y radiológicas.

Los esteroides son la piedra angular del tratamiento de la ABPA, la bibliografía recomienda regímenes más agresivos para inducir la remisión. Los antifúngicos, como el itraconazol, se recomiendan utilizar como ahorrador de esteroides o cuando ha habido recaída pese a una terapia inicial con esteroides.

\section{BIBLIOGRAFÍA}

1. Manuel Soto-Martínez, Manuel SotoQuiros. Epidemiología del asma en Costa Rica. Rev. Méd. Hosp. Nac. Niños 2004; 39: 42-53.

2. Moss R, Treatment options in severe fungal asthma and allergic bronchopulmonary aspergillosis. Eur Respir J 2014; 43: 1487-1500

3. Nicholas E. Vlahakis, Timoathy $R$ Aksamit. Diagnosis and Treatment of Allergic Bronchopulmonary Aspergillosis. Mayo Clin Proc, 2001; 76: $930-938$

4. Agarwal Ritesh. Allergic Bronchopulmonary Aspergillosis. CHEST 2009, 135(3), 805-826.

5. Karen Patterson, Mary E. Strek Allergic Bronchopulmonary Aspergillosis. Proc Am Thorac Soc 2010 7: 237-244

6. Paul A. Greenberger. When to suspect and work up allergic bronchopulmmonary aspergillosis. Ann Allergy Asthma Immunol 2013, 1-4.

7. Agarwal R, Chakrabarti A, Shah A Agarwal R, Chakrabarti A, Shah A y col Allergic bhroncopulmonar aspergillosis: review of literature and proposal of new diagnostic and classification criteria. Clinical \& Experimental Allergy 43 850-873.

8. Pla Villalobos, Jonathan. Prevalencia de Hipersensibilidad a Antígenos de Aspergillus sp en Asmáticos Severos del Hospital San Juan de Dios Setiembre 2010 a Febrero 2011. Tesis de Especialidad Médica, Universidad de Costa Rica, San José, Costa Rica.

9. Alfred P. Fishman, Jack A. Elias, Jay A. fishman y col. Fishman's Pulmonary Diseases and Disorders. Cuarta Edición Mc-Graw Hill Medical, Estados Unidos, Capítulo 49. 837-844

\section{DECLARACIÓN DE CONFLICTO DE INTERESES}

Los autores declaran que no existen conflictos de intereses. 